\title{
Analytical BER and PER Performance of Frequency-Domain Diversity Combining, Multipacket Detection and Hybrid Schemes
}

\author{
Francisco Ganhão, Student Member, IEEE, Rui Dinis, Member, IEEE, \\ Luis Bernardo, Member, IEEE, and Rodolfo Oliveira, Member, IEEE
}

\begin{abstract}
Diversity Combining (DC) and Multipacket Detection (MPD) are efficient techniques that cope with packet errors due to severe propagation conditions and/or collisions. To evaluate their network performance on a network simulator, an accurate characterization of the Bit Error Rate (BER) and Packet Error Rate (PER) can successfully replace the behavior of the PHY layer. This choice is better in terms of computational time than implementing a full PHY layer to be jointly simulated with the upper layers. In this paper, an analytical model is proposed to compute the BER and PER of DC, MPD and hybrid techniques, considering a Single-Carrier with Frequency-Domain Equalization (SC-FDE) scenario. The paper also considers two frequency-domain detection methods: a simple linear receiver and a powerful iterative receiver. The proposed model is simple and versatile, since it can support nonhomogeneous transmitting powers, and different channel conditions concerning retransmissions and channel randomization techniques. Several simulations show that the proposed model provides accurate BER and PER results for a wide range of scenarios.
\end{abstract}

Index Terms-SC-FDE, IB-DFE, multipacket detection, diversity combining, hybrid $\mathrm{ARQ}$, analytical performance.

\section{INTRODUCTION}

$\mathbf{F}$ UTURE wireless broadband systems are expected to have high transmission rates with high throughput, low delay and high power efficiency. The multipath propagation channel can be strongly selective, due to high transmission rates, leading to severe time-distortion effects. Block transmission techniques combined with frequency-domain processing are appropriate for high transmission rates over severely timedispersive channels. Orthogonal Frequency Division Multiplexing (OFDM) [1] is the most popular modulation based on this concept. However, the high envelope fluctuations of

Paper approved by H. Leib, the Editor for Communication and Information Theory of the IEEE Communications Society. Manuscript received July 7, 2011; revised November 14, 2011 and February 11, 2012.

F. Ganhão is with the FCT-UNL - Universidade Nova de Lisboa, Instituto de Telecomunicações and UNINOVA, Portugal (e-mail: fjsg@campus.fct.unl.pt).

R. Dinis is with the FCT-UNL - Universidade Nova de Lisboa and Instituto de Telecomunicações, Portugal (e-mail: rdinis@fct.unl.pt)

L. Bernardo and R. Oliveira are with the FCT-UNL - Universidade Nova de Lisboa and UNINOVA, Portugal (e-mail: $\{1 \mathrm{flb}$, rado $\} @$ fct.unl.pt).

This work was partially published in IEEE VTC Spring 2011 and supported to a certain extent by the FCT/MEC (CTS multi-annual funding project PEst-OE/EEI/UI0066/2011, IT pluri-annual funding project PEst-OE/EEI/LA0008/2011, MPSat project PTDC/EEA-TEL/099074/2008, OPPORTUNISTIC-CR project PTDC/EEA-TEL/115981/2009 and grant SFRH/BD/66105/2009).

Digital Object Identifier 10.1109/TCOMM.2012.061212.110440
OFDM signals are not recommendable for the uplink transmission, especially with highly efficient power amplification at the mobile terminals. Single-Carrier with Frequency-Domain Equalization (SC-FDE) schemes [2] are adequate for this case under severely time-dispersive channels, with much lower envelope fluctuations than OFDM [3], [4]. The performance of a conventional SC-FDE can be further improved if the linear FDE is replaced by an Iterative Block Decision Feedback Equalization (IB-DFE) [5]. For these reasons, SC-FDE schemes were selected for the uplink of wireless broadband systems [6].

Transmitted packets can be lost because of poor transmission conditions, e.g., high noise or interference levels, or collisions. Usually a packet with errors is discarded and henceforth retransmitted. However, the information in the signal associated to a failed transmission attempt (i.e., an erroneous packet) could be used to enhance data reception. For this reason several techniques were proposed to cope with lost packets. These techniques combine all packet transmission atempts to recover the original packet, instead of simply discarding a packet with errors.

If a packet was lost due to noise or interference, HybridARQ (HARQ) techniques can be employed to enhance data reception [7]. HARQ techniques can be loosely arranged in two categories: Code Combining (CC) and Diversity Combining (DC) systems. In CC systems [8], [9], multiple copies of the same packet are concatenated to form noise-corrupted codewords with increasingly longer codewords and lower rate codes. In DC systems (e.g. [10]), the individual symbols from identical copies of a packet are combined to create a single packet with more reliable symbols. DC systems are generally suboptimal with respect to CC systems, but are simpler to implement [7]. DC techniques are easily extended to SC-FDE schemes [11]; these techniques allow significant improvements in terms of delay and throughput performance [12].

However, HARQ techniques are not appropriate when multiple Mobile Terminals (MTs) access the channel because in those cases, the traditional Medium Access Control (MAC) approach to avoid collisions is to ask packet retransmissions from MTs with different probabilities. As an alternative, MultiPacket Detection (MPD) [13] techniques could be employed.

There are several MPD approaches [14]-[16]. This article considers MPD techniques based on time diversity, such as the one employed in the Network Diversity Multiple Access 
(NDMA) context [17]. The basic idea behind time diversity MPD is that all MTs involved in a collision transmit several copies of their packets under slightly modified transmission conditions in such a way that the receiver, i.e. the BS, is able to separate all packets involved in the collision. To allow efficient packet separation, the subsequent retransmissions should be made with almost uncorrelated channels. When this is not practical, it is possible to employ different packet phase rotations for different MTs [17] or interference cancelation procedures [18]. For severely time-dispersive channels combined with frequency-domain receivers, it is possible to take advantage of the high variability of the channel frequency response. For an efficient packet separation, cyclic shifts [19] or frequency-domain scrambling [20] could be performed on the retransmitted packets. When employed in the DC context, these techniques also provide additional diversity effects [11].

The study of DC and MPD at the network level is difficult, since there are no closed formulas for the Bit Error Rate (BER) and Packet Error Rate (PER). In most studies, average BER and PER values are previously obtained from extensive physical layer simulations and then used by a network simulator, usually under specific conditions, e.g., perfect power control. Another option would be to implement the physical layer in the network simulator, although this would significantly increase the complexity and duration of network simulations. A better solution in terms of computation would be to have simple formulas of the BER and PER for a given scenario and employ them in a network simulator.

This paper considers SC-FDE schemes where DC and MPD techniques are employed to cope with lost packets. Two frequency-domain equalization techniques are also considered: linear equalization, and nonlinear iterative equalization based on the IB-DFE concept. The main objective of the paper is to provide an accurate and simple analytical model to obtain the BER and PER performance for DC and time-diversity MPD. The proposed model is employed in a wide range of DC and MPD scenarios, as well as hybrid schemes that combine DC and MPD principles.

Theoretical bounds on the performance of uncoded OFDM and SC-FDE schemes were obtained in [21] and exact formulas for specific channels were derived in [22]. Assuming a Gaussian characterization of the signals at the FDE output, it is possible to obtain simple BER performance formulas [23], [24].

Regarding the notation adopted in this work, $\mathbf{A}$ is used to denote a matrix, $\mathbf{A}^{T}$ is the matrix transpose of $\mathbf{A}, \mathbf{A}^{H}$ is the complex conjugate transpose of $\mathbf{A}$ and $\mathbf{A}^{*}$ is the complex conjugate of $\mathbf{A}$.

The structure of the paper is as follows: Section II characterizes the proposed system and Section III analyzes the receiver structures for DC and MPD and provides an analytical model for their performance evaluation. Section IV refers to alternative retransmission techniques that can be studied with the proposed model. Section V studies the performance of the analytical model in a wide range of scenarios. Finally, Section VI briefly comments the paper's conclusions.

\section{SYSTEM CHARACTERIZATION}

This work considers a structured wireless system employing SC-FDE at the uplink, where a set of Mobile Terminals (MTs) send data to a Base Station (BS) using a slotted data channel. MTs are low resource battery operated devices, whereas the BS is a high resource device that can use DC to cope with packet errors due to poor propagation conditions or MPD due to collisions. The BS can also employ hybrid techniques that combine DC and MPD. The MTs send data packets on time slots defined by the BS (for the sake of simplicity, it is assumed that the packets associated to each MT have the same duration and a packet corresponds to a time slot). Perfect channel estimation and synchronization is assumed. It is also assumed that colliding packets on each slot arrive simultaneously, which means that perfect time advance mechanisms exist to compensate different propagation times.

Multiple MTs may transmit packets during each slot. To resolve possible collisions, the slots in the uplink channel are organized into epochs. An epoch is defined as a set of slots that a group of MTs use to concurrently transmit packets. It is assumed that the BS is capable of detecting the number of MTs, $P$, transmitting in a given channel during a slot. During an epoch where $P$ MTs transmit, there are at most $P$ MTs transmitting in each slot. A maximum duration of slots, $L_{\max }$, is usually defined for the epoch. Furthermore, the BS may run the detection algorithm at the end of each slot of an epoch to acknowledge the successfully received packets, and only request additional retransmissions of the unsuccessfully received ones. In a Local Area Network (LAN), this feedback is almost instantaneous allowing an Hybrid Automatic Repeat reQuest (H-ARQ) approach; however, for a network with a measurable Round Trip-Time (RTT) (e.g. where the BS is a satellite or a high-altitude platform) the acknowledgement will take longer to arrive. Therefore, the actual duration of an epoch will depend on the kind of network and respective conditions. The $p$ th MT involved in a collision may retransmit its data packet $L_{p}-1$ times during an epoch, where different $L_{p}$ values may be used for MTs with different signal to noise ratios. The epoch duration is defined by the largest $L=\max \left\{L_{p}, p=1 . . P\right\}$. At the end of an epoch, the BS signals whose data blocks that were successfully decoded and received.

In MPD for NDMA [17], [19], it was shown that a minimum of $L=P$ packet transmissions are required when $P$ MTs are involved in a collision with perfect constant average power control at the reception. But the BS may also signal MTs to retransmit their data $L>P$ times. Such case arises when a combination of MPD with DC technique is employed. On the instance that $P=1$ and $L>P$, it can be classified as DC, since only one MT is transmitting data for $L>1$. Considering that $P$ MTs access simultaneously the channel with different attenuation gains $\left|\xi_{v}\right|$, with $v$ ranging from 1 up to a maximum of $N_{\text {Gain }}$, the BS can ask fewer retransmissions than $P$. Considering an IB-DFE technique that performs Successive Interference Cancellation (SIC) for each iteration, it allows the reception of more than one packet per slot in average [25]. Using the IB-DFE receiver proposed in [26], MTs near and far away from the BS can coexist in the same slots, due to 